\title{
CRECIMIENTO DE Eucalyptus camaldulensis DEHNH. EN RELACIÓN A ATRIBUTOS EDAFOAMBIENTALES A NIVEL DE MICROSITIO EN LUJÁN, BUENOS AIRES, ARGENTINA
}

\author{
Craig, Elena ${ }^{6}$ y Momo, Fernando ${ }^{7}$
}

\section{RESUMEN}

En Argentina, las plantaciones de Eucalyptus spp. representan el $32 \%$ del total de plantaciones y el $27,7 \%$ del total de materia prima en la industria forestal (Sánchez Acosta y Vera, 2005).

Eucalyptus camaldulensis es la especie cultivada de mayor aptitud en todo el país por su gran plasticidad; resiste condiciones de extrema sequía, tolera suelos salinos y se adapta a subsuelos con terreno arcilloso.

Si bien se ha realizado evaluaciones de calidad de sitio de E. camaldulensis en Argentina (Baridón et al ,2001), no se ha encontrado trabajos que vinculen los atributos edáficos con el crecimiento a nivel individual de rodales y el efecto de los mismos en la productividad y heterogeneidad del rodal.

Asimismo, es importante en el inventario de un rodal, relacionar el volumen, área basal y DAP con los atributos edáficos (Correa Neto et al, 2007). El objetivo del trabajo es evaluar los atributos edafoambientales a nivel de micrositio y su relación con el crecimiento de los árboles y la heterogeneidad del rodal.

El crecimiento de Eucalyptus camaldulensis fue medido en árboles plantados en dos rodales localizados en Jauregui, Lujan, Buenos Aires, Argentina. La altura y el diámetro a la altura del pecho (DAP) fueron medidos anualmente en 100 árboles el tercero, cuarto y quinto año de plantación de los rodales.

La densidad de plantación es de 1111 arb/ha a un espaciamiento de $3 \times 3 \mathrm{~m}$. Al mismo tiempo, se evaluó atributos químicos y físicos del suelo a nivel del micrositio alrededor de cada árbol. Estos atributos fueron profundidad del primer horizonte (Horizonte A), del segundo horizonte (Horizonte B) y del tercer horizonte (Horizonte arcilloso $B_{t}$ ); contenido de materia orgánica, contenido de fósforo disponible y $\mathrm{pH}$.

Los datos fueron analizados usando análisis multivariado discriminante (DA) y luego corroborados mediante regresión simple y múltiple de variables dendrométricas versus atributos edafoambientales.

Se clasificó los árboles en cuatro categorías de acuerdo a su altura total, dividiendo los datos en cuartiles. Los árboles incluidos en los cuartiles 1, 2, 3 y 4 fueron categorizados como dominados, intermedios, co-dominantes y Dominantes, respectivamente.

\footnotetext{
${ }^{6}$ Depto. de Tecnología. Universidad Nacional de Lujan, Argentina. craigelena@yahoo.com.ar

${ }^{7}$ Depto. de Cs. Bs., UNLu e Instituto de Ciencias de la Universidad Nacional de General Sarmiento, Argentina. fmomo@ungs.edu.ar
} 
El mismo procedimiento se realizó con el DAP y el volumen. El análisis multivariado discriminante concluye que el primer eje canónico explica el $71,19 \%$ de la variación entre grupos. La primera función discriminante puede expresarse de la siguiente manera:

$$
\mathrm{F}=0.19^{*} \text { prof } 1+0.29^{*} \text { prof } 2+0.31^{*} \% \text { C.O. }+0.11^{*} \text { fósforo }+0.38^{*} \mathrm{pH} 1-.19^{*} \mathrm{pH} 2+0.85^{*} \mathrm{pH} 3 \text {. }
$$

El pH del horizonte $B_{t}$ es la variable más importante para la discriminación sobre el primer eje canónico. Los árboles dominantes están agrupados solo en el área izquierda en el primer eje canónico, asociados a $\mathrm{pH}$ del horizonte $\mathrm{B}_{\mathrm{t}}$ más bajos.

Similares resultados se obtuvo analizando el DAP y el volumen. Analizando todos los árboles con sus categorías sociales, se puede observar que la altura total presentó una regresión lineal $\left(r^{2}=0,33\right)$ respecto al $\mathrm{pH}$ del horizonte $B_{t} \quad(p<0,0001)$, pero que dicha regresión mejoró notablemente $\left(r^{2}=0.62\right)$, cuando solo se usan en el análisis los árboles dominantes.

El pH del suelo fue la variable más significativa en relación al crecimiento de los árboles. Se encontró que los árboles dominantes crecieron en los micrositios con menores pH del horizonte $B_{t}$. Las regresiones de la altura total y el $\mathrm{pH}$ del horizonte $\mathrm{B}_{\mathrm{t}}$ mejoraron notablemente usando en las mismas, solo la categoría social correspondiente a los árboles dominantes.

Palabras clave: Eucalyptus camaldulensis, calidad micrositio 


\section{SUMMARY}

In Argentina, Eucalyptus plantations represent $32 \%$ of total plantings and $27.7 \%$ of total raw material in the forest industry (Sanchez Acosta and Vera, 2005).

Eucalyptus camaldulensis is the most cultivated species for its great plasticity; resists extreme drought, saline soils and tolerates clay subsoils.

While assessments were made in E. camaldulensis site quality in Argentina (Baridon et al, 2001), no information has been found that link soil attributes with individual-level growth of stands and their effect on productivity and heterogeneity of the stand. It is also important in the inventory of a stand, relate the volume, basal area and DAP, with soil attributes (Correa Neto et al, 2007).

The objective of this study is to evaluate the microsite factors and its relation to tree growth and stand heterogeneity.

The growth of Eucalyptus camaldulensis trees was measured in two stands located in Jauregui, Lujan, Buenos Aires, Argentina. The height and breast height diameter (DBH) were measured in 100 trees annually, the third, fourth and fifth year of plantation stands. Planting density was 1111 plants/ha at a spacing of $3 \times 3 \mathrm{~m}$.

At the same time, the chemical and physical soil attributes were evaluated around each tree. These attributes were depth of the first horizon (Horizon A), the second horizon (Horizon B) and third horizon (Bt horizon clay), organic matter content, available phosphorus content and $\mathrm{pH}$.

Data were analyzed using multivariate discriminant analysis (DA) and then corroborated by simple and multiple regression of dendrometric variables versus soil factors.

Trees were classified into four categories according to their full height, dividing the data into quartiles. The trees included in quartiles $1,2,3$ and 4 were categorized as dominated, intermediate, co-dominant and dominant, respectively. The same procedure was performed with the $\mathrm{DBH}$ and volume.

Multivariate discriminant analysis concludes that the first canonical axis explains $71.19 \%$ of the variation between groups. The first discriminant function can be expressed:

$\mathrm{F}=0.19^{*}$ depth $+0.29^{*}$ depth $2+0.31^{*} \% \mathrm{CO}+0.11^{*}$ phosphorus $+0.38^{*} \mathrm{pH} 1-.19^{*} \mathrm{pH} 2+0.85^{*} \mathrm{pH} 3$.

The $\mathrm{pH}$ of the $\mathrm{Bt}$ horizon was the most important variable for discrimination on the first canonical axis. The dominant trees are grouped only in the area left at the first canonical axis, associated with $\mathrm{pH}$ lower Bt horizon. Similar results were obtained by analyzing the $\mathrm{DBH}$ and volume. 
Analyzing all the trees with their social categories, it can be found that the total height presented a linear regression $(\mathrm{r} 2=0.33)$ with the Bt horizon $\mathrm{pH}(\mathrm{p}<0.0001)$, but significantly improved the regression ( $r 2=0.62)$, when only used the dominant trees.

Soil $\mathrm{pH}$ was the most significant variable in relation to tree growth. It was found that the dominant trees grew in microsites with lower $\mathrm{pHs}$. The Bt horizon regressions of total height and $\mathrm{pH}$ of the Bt horizon greatly improved using only the social category corresponding to the dominant trees.

Key words: Eucalyptus camaldulensis, Quality microsite 


\section{INTRODUCCIÓN}

La productividad forestal está regulada por una combinación de factores ambientales, incluyendo radiación, temperatura, agua y disponibilidad de nutrientes (Binkley, 1993).

La calidad de sitio forestal expresa la productividad potencial de un rodal y puede ser evaluada directamente mediante mediciones dasométricas que expresan las influencias ambientales y biológicas en el crecimiento de un árbol o indirectamente a través de estimaciones realizadas por mediciones de atributos del sitio que expresan esa capacidad (Carmean, 1975).

La calidad de sitio puede variar sustancialmente en unos pocos metros, generando heterogeneidad en el crecimiento de los árboles y afectando la productividad del rodal.

Las plantaciones de Eucalyptus en Argentina representan actualmente el 32\% del total del plantaciones y el $27.7 \%$ del total del material industrializado (Sánchez Acosta y Vera, 2005). La especie Eucalyptus camaldulensis se ha empleado en forestación históricamente en la provincia de Buenos Aires con fines de sombra y reparo, como parte de cortinas y macizos (Borzone et al, 2007). Es una especie extremadamente plástica, si bien en suelos con limitaciones el crecimiento y productividad, así como la forma de la misma, se ven restringidas (FAO, 1956, Moschini et al., 2000).

Dado que Argentina presenta potencialidad forestal en sitios de uso actual agropecuario y que se desconoce en dichos suelos cuales son los atributos que influyen en el crecimiento de Eucalyptus camaldulensis Dehnh, es importante evaluar los rodales existentes, a nivel de micrositio, como herramienta de predicción de modelos de productividad.

\section{OBJETIVOS}

Estimar la respuesta del crecimiento de individuos de Eucalyptus camaldulensis a diferentes condiciones de micrositio.

Describir la heterogeneidad de crecimiento de los árboles y su relación con la heterogeneidad de micrositios.

Estimar la importancia relativa de diferentes características y propiedades edáficas en relación al crecimiento individual de los árboles.

\section{MATERIALES Y MÉTODOS}

\section{Lugar de Ensayo y Caracterización del Sitio}

El estudio se realizó en dos rodales correspondientes a plantaciones comerciales de Eucalyptus camaldulensis, durante el tercer, cuarto y quinto año de edad, plantados con el propósito de obtener materia prima para paneles de partículas.

Estos rodales estaban ubicados en la ciudad de Jáuregui, Lujan, Argentina, a $34^{\circ} 35^{\prime} \mathrm{LS}$ y $59^{\circ}$ LO. La densidad de plantación fue de 1.111 plantas por hectárea, su espaciamiento fue de 3 x $3 \mathrm{~m}$ y las mismas fueron obtenidas de semilla local. La superficie de cada rodal fue de 10 ha, dentro de las cuales se seleccionó al azar 100 árboles. Se evaluó parámetros dendrométricos en 100 plantas y atributos edáficos en los sitios alrededor de las mismas. 
El sitio tiene una pendiente del 0-2\%. El clima es templado húmedo con una temperatura media anual de $16,4^{\circ} \mathrm{C}$. La precipitación media anual es de $938,5 \mathrm{~mm}$ con un pico de lluvias en otoño. Los suelos muestran déficit hídrico solo durante los meses de diciembre y enero (verano).

El paisaje exhibe una gran heterogeneidad topográfica, mostrando micro depresiones. Las parcelas están localizadas en el plano aluvial del Río Luján y sufren inundaciones temporarias. El suelo es un Argiudol Típico, arcilloso, fino, ilítico y térmico.

Los árboles evaluados fueron elegidos al azar dentro de la plantación. Se midió altura con clinómetro Suunto, diámetro a la altura del pecho (DAP) con cinta diamétrica, proyección de la copa, presencia de bifurcaciones, altura de bifurcaciones y número de ejes.

Debido a la heterogeneidad horizontal y vertical de estos suelos, y a la presencia de un horizonte $B_{t}$ arcilloso, se tomaron muestras detalladas $(25 \mathrm{~cm} \times 25 \mathrm{~cm} \times 25 \mathrm{~cm})$ a una distancia de un metro ubicado al azar alrededor de cada árbol. Las muestras fueron tamizadas a través de un tamiz de $2 \mathrm{~mm}$. La fracción fina del suelo $(<2 \mathrm{~mm})$ fue utilizada para los análisis químicos.

Se evaluó de cada micrositio el espesor del horizonte superficial, subsuperficial y la profundidad hasta el horizonte $\mathrm{B}_{\mathrm{t}}$, los $\mathrm{pH}(1: 2,5)$ en todos los horizontes y en el horizonte superficial, además se evaluó el contenido de materia orgánica (Walkley-Black) y el contenido de fósforo disponible (Kurtz and Bray II).

En una submuestra de 17 árboles dominantes con sus respectivas muestras de suelo, se evaluó además el contenido de nitrógeno total (Kjendal), la relación $\mathrm{C} / \mathrm{N}$, la conductividad eléctrica (mmhos/cm), el contenido de Calcio (EDTA), el contenido de Magnesio (EDTA), el contenido de Potasio (fotómetro de llama), el contenido de sodio (fotómetro de llama) y la densidad aparente $\left(\mathrm{g} / \mathrm{cm}^{3}\right)$ del horizonte superficial.

\section{Análisis Estadístico}

Se clasificó los árboles en cuatro categorías de acuerdo a su altura total, dividiendo los datos en cuartiles y dando un código numérico a cada árbol de acuerdo al cuartil al cual pertenece. Un procedimiento similar se realizó para el DAP (diámetro a la altura del pecho) y el VOL (Volumen real con corteza). Luego se realizó un análisis multivariado discriminante, usando los códigos de los cuartiles como criterio de clasificación. Los árboles incluidos en los cuartiles 1, 2, 3 y 4 fueron categorizados como dominados, intermedios, co-dominantes y dominantes, respectivamente.

A partir de la selección de atributos edáficos de mayor significancia sobre las variables dendrométricas, se realizó regresiones múltiples. Las mismas se hicieron con la totalidad de los árboles y con los árboles dominantes solamente. Además, se realizaron en la submuestra de árboles dominantes con mayor número de variables edáficas. En todos los casos se utilizó el programa estadístico INFOSTAT ${ }^{\circledR}(2009)$ y el XLSTAT ${ }^{\circledR}(2010)$.

\section{Atributos de Crecimiento}

Respecto de la altura total, se asume que el crecimiento en altura total no es afectado por la densidad del rodal y por lo tanto es un excelente indicador de la calidad de sitio e íntimamente relacionado con el sitio y la genética.

En el caso del DAP, si bien se analizó su relación con los atributos edáficos, estas relaciones dependerán del nivel de competencia de los rodales. 


\section{RESULTADOS}

\section{Relación Suelo - Planta a Nivel de Micrositio y Categorías Sociales}

Los valores de las variables dendrométricas por cuartiles fueron clasificados en las cuatro categorías de árboles (dominados, intermedios, co-dominantes y dominantes) respecto de cada atributo dendrométrico, siendo los árboles dominados aquellos comprendidos entre el valor mínimo y el primer cuartil de la variable, los intermedios aquellos comprendidos entre el primer cuartil y la mediana, los co-dominantes comprendidos entre la mediana y el tercer cuartil y los dominantes aquellos comprendidos entre el tercer cuartil y el valor máximo de la variable de clasificación (Cuadro $\mathrm{N}^{\circ} 1$ ).

\section{Cuadro $\mathrm{N}^{\circ} 1$}

CARACTERIZACIÓN DE VARIABLES DENDROMÉTRICAS PARA LAS CUATRO CATEGORÍAS DE ÁRBOLES AL TERCER AÑO DE EDAD

\begin{tabular}{|l|c|c|c|c|}
\hline \multicolumn{1}{|c|}{ Variable } & $\begin{array}{c}\text { Dominados } \\
\text { ( Código 1) }\end{array}$ & $\begin{array}{c}\text { Intermedios } \\
\text { ( Código 2) }\end{array}$ & $\begin{array}{c}\text { Co-Dominantes } \\
\text { (Código 3) }\end{array}$ & $\begin{array}{c}\text { Dominantes } \\
\text { ( Código 4) }\end{array}$ \\
\hline Altura $(\mathrm{m})$ & $1,18-2,46$ & $2,46-3,21$ & $3,21-4,61$ & $4,61-6,80$ \\
\hline DAP $(\mathrm{cm})$ & $0,32-3,18$ & $3,18-5,73$ & $5,73-10,83$ & $10,83-17,20$ \\
\hline Volumen $\left(\mathrm{m}^{3}\right.$ /árbol) & $4,74266 \mathrm{E}-06-0,00097$ & $0,00097-0,0041$ & $0,0041-0,021$ & $0,021-0,078$ \\
\hline
\end{tabular}

\section{Análisis Multivariado Discriminante en Relación a Altura total al Tercer Año}

En primer lugar se realizó un análisis de multicolinearidad entre variables de sitio, para evitar aquellas cuyo rango de tolerancia no eran admitidas por el modelo. Todas las variables evaluadas entraron en el modelo. En la Figura $\mathrm{N}^{\circ} 1$ se muestra la separación de cuatro grupos de acuerdo a las primeras dos raíces discriminantes. El atributo edáfico significativo estadísticamente en el primer eje discriminante es el pH del $\mathrm{B}_{\mathrm{t}}$. (Cuadros $\mathrm{N}^{\circ} 2, \mathrm{~N}^{\circ} 3$ y $\mathrm{N}^{\circ} 4$ ).

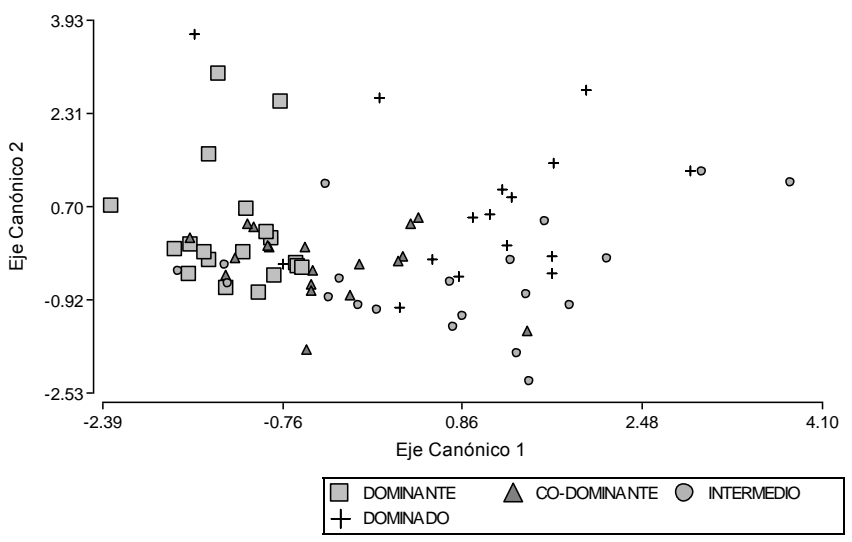

Figura $\mathrm{N}^{\circ} 1$

ANÁLISIS DISCRIMINANTE DE LA ALTURA AL TERCER AÑO DE PLANTACIÓN 
CUADRO $\mathrm{N}^{\circ} 2$

RESUMEN DE LAS FUNCIONES CANÓNICAS DISCRIMINANTES

\begin{tabular}{|c|r|r|r|}
\hline Función & Autovalor & \multicolumn{1}{|c|}{$\begin{array}{c}\text { Varianza } \\
(\%)\end{array}$} & $\begin{array}{c}\text { Acumulado } \\
(\%)\end{array}$ \\
\hline 1 & 0,80 & 71,19 & 71,19 \\
\hline 2 & 0,26 & 22,82 & 94,00 \\
\hline 3 & 0,07 & 6,00 & 100,00 \\
\hline
\end{tabular}

Cuadro $\mathrm{N}^{\circ} 3$

RESUMEN ANÁLISIS FUNCIÓN DISCRIMINANTE

\begin{tabular}{|l|c|c|cc|}
\hline \multicolumn{1}{|c|}{ Variable } & Wilks & Parcial & \multicolumn{2}{c|}{ F Remove } \\
& Lambda & Lambda & (2.56) & Nivel p \\
\hline Profundidad A & 0.548131 & 0.964687 & 0.841923 & 0.475571 \\
Profundidad B & 0.529760 & 0.998140 & 0.042849 & 0.988096 \\
\% Carb. Org. & 0.532774 & 0.992494 & 0.173952 & 0.913669 \\
Fósforo & 0.534862 & 0.988620 & 0.264763 & 0.850558 \\
pH horizonte A & 0.595497 & 0.887957 & 2.902169 & $\mathbf{0 . 0 4 0 9 7 2}$ \\
pH horizonte B & 0.582551 & 0.907689 & 2.339071 & 0.080998 \\
pH horizonte Bt & 0.669491 & 0.789817 & 6.120682 & $\mathbf{0 . 0 0 0 9 4 1}$ \\
\hline
\end{tabular}

Cuadro $\mathrm{N}^{\circ} 4$

RESUMEN DE LA FUNCIÓN DISCRIMINANTE EN RELACIÓN A LA ALTURA

\begin{tabular}{|l|r|r|}
\hline \multicolumn{1}{|c|}{ Variable } & \multicolumn{1}{|c|}{$\begin{array}{c}\text { Matriz } \\
\text { Estructura }\end{array}$} & $\begin{array}{c}\text { Coeficientes } \\
\text { Estandarizados }\end{array}$ \\
\hline Constante & $-16,98$ & 0,19 \\
Profundidad A & 0,03 & 0,29 \\
Profundidad B & 0,03 & 0,31 \\
\% Carb. Org. & 1,03 & 0,11 \\
Fósforo & 0,04 & 0,38 \\
pH horizonte A & 0.90 & $-0,19$ \\
pH horizonte B & $-0,34$ & $\mathbf{0 , 8 5}$ \\
pH horizonte Bt & 1,50 & \\
\hline
\end{tabular}

El análisis de los autovalores concluye en que el primer eje canónico explica el $71,19 \%$ de la variación entre grupos (Cuadro $\mathrm{N}^{\circ} 2$ ). 
La primera función discriminante puede expresarse de la siguiente manera:

$$
\mathrm{F}=0.19^{*} \text { prof } 1+0.29^{*} \text { prof } 2+0.31^{*} \% \text { C.O. }+0.11^{*} \text { fósforo }+0.38^{*} \mathrm{pH} 1-0.19^{*} \mathrm{pH} 2+0.85^{\star} \mathrm{pH} 3
$$

Los valores de $\mathrm{pH}$ del horizonte $\mathrm{B}_{\mathrm{t}}$ entre 6 y 6,7 están asociados a la presencia de árboles dominantes. Los árboles intermedios y dominados están presentes en micrositios con $\mathrm{pH}$ del horizonte $B_{t}$ entre 6,8 y 8,3 . El límite para los árboles Co-dominantes es de un $\mathrm{pH}$ del horizonte $\mathrm{B}_{\mathrm{t}}$ de 6,4 a 7,6 .

En la Figura $\mathrm{N}^{\circ} 2$ se muestra la variación del $\mathrm{pH}$ del horizonte $\mathrm{B}_{\mathrm{t}}$ por categorías sociales de árboles, encontrándose que los árboles dominantes se encuentran asociados a pH del horizonte $B_{t}$ más bajos y con un rango menos amplio, lo que indicaría que los árboles dominantes se encuentran en sitios de menor pH y son más homogéneos en su distribución respecto a este parámetro edáfico.

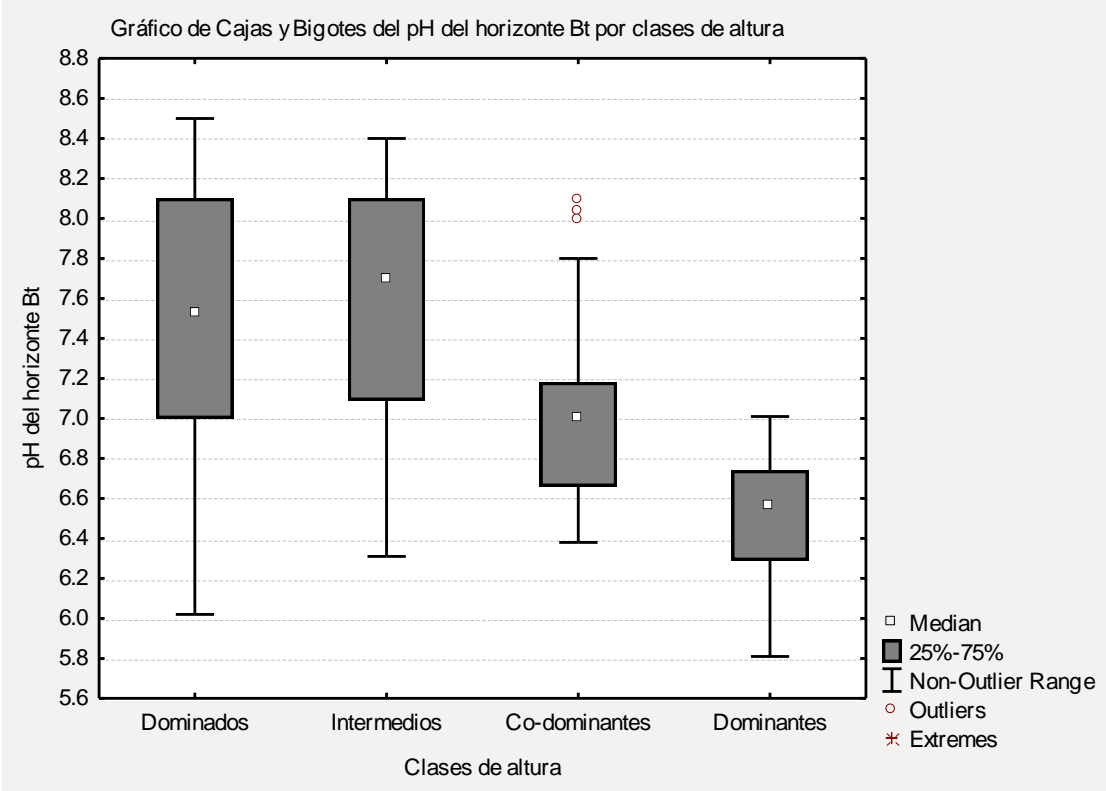

\section{GRÁFICO DE CAJAS Y BIGOTES DEL pH DEL HORIZONTE $B_{t}$ POR CLASES SOCIALES DE ALTURA}

El mismo análisis discriminante fue realizado para el DAP y para el volumen como variables dependientes. Dado que la relación alométrica entre altura y DAP con un $R^{2}=0,94$ los resultados de ambos análisis mostraron situaciones equivalentes. Además, este análisis se realizó para el cuarto y quinto año de edad, coincidiendo lo encontrado con el tercero. 


\section{Regresión Lineal Altura Total Versus Aributos Edáficos en Ambos Rodales en Todas las Categorías de Árboles}

Se realizó una regresión lineal múltiple de los atributos edáficos versus la altura total, tomando esta variable como indicadora de la calidad de sitio.

Como puede observarse en la Figura $\mathrm{N}^{\circ} 3$, existe una relación lineal entre la altura total y el $\mathrm{pH}$ del horizonte $\mathrm{B}_{t}(\mathrm{p}<0,0001)$. Tomando la información de los coeficientes de regresión se llega a la siguiente ecuación para los rangos de $\mathrm{pH}$ del modelo:

$$
Y=a+b x=14,91-1,07^{*} p H \text { horizonte } B_{t}
$$

El valor del coeficiente de determinación $\left(R^{2}=0,42\right)$ y la significación del contraste $F$ global de la regresión $(p<0,0001)$ sugieren que parte de la variabilidad de la respuesta está explicada por el modelo ajustado. Existe una relación lineal negativa entre la altura total al tercer año y el $\mathrm{pH}$ del horizonte $\mathrm{B}_{\mathrm{t}}$.

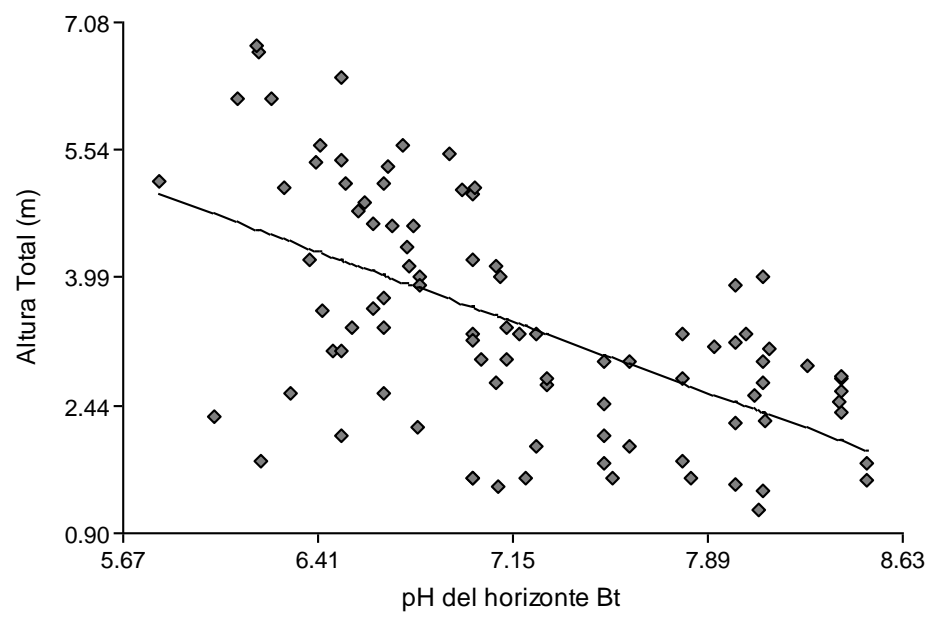

Figura $\mathrm{N}^{\circ} 3$

DISPERSIÓN DE REGRESIÓN LINEAL DE ALTURA TOTAL VERSUS pH DEL HORIZONTE $\mathrm{B}_{\mathrm{t}}$

\section{Regresión Lineal de Altura Total Versus el Total de Atributos Edáficos en Ambos Rodales en la Categoría Social de Árboles Dominantes}

La profundidad del horizonte A (prof 1), la conductividad eléctrica (CE), el pH del horizonte $\mathrm{B}_{\mathrm{t}}(\mathrm{pH} 3)$ y el contenido de Magnesio del suelo presentan una relación lineal significativa $(p<0,10)$. Tomando la información de los coeficientes de regresión se llega a la siguiente ecuación:

$$
\mathrm{Ht}=25,94-0,12^{*} \text { prof } \mathrm{A}-14,17{ }^{*} \mathrm{CE}-1,89 * \mathrm{pH} \mathrm{B}+5,61{ }^{*} \mathrm{Mg}
$$


El valor del coeficiente de determinación $\left(R^{2}=0,91\right)$ y la significación del contraste $T$ global de la regresión $(p<0,10)$ sugieren que gran parte de la variabilidad de la respuesta está explicada por el modelo ajustado. En la Figura $N^{\circ} 4$ se muestra los gráficos de los residuos parciales en los que se observa que:

Existe una relación lineal positiva entre la altura total al tercer año y la profundidad del horizonte A.

Existe una relación lineal negativa entre la altura total y la CE del suelo.

Existe una relación lineal negativa entre la altura total y el pH del horizonte $\mathrm{B}_{\mathrm{t}}(\mathrm{pH} 3)$.

Existe una relación lineal positiva entre la altura total y el contenido de Magnesio del suelo.

Concluyendo, en la regresión lineal múltiple de la altura total versus variables edáficas se mejoró su capacidad predictiva cuando se uso solo la categoría social de árboles dominantes $\left(R^{2}=0,42\right.$ versus $\left.R^{2}=0,91\right)$.

El mismo análisis de regresión lineal fue realizado para el DAP y para el volumen como variables dependientes. Dado que la relación alométricas entre altura y DAP presentaron un $\mathrm{R}^{2}=0,95$ para esta submuestra de árboles dominantes, los resultados de ambos análisis mostraron situaciones equivalentes. 

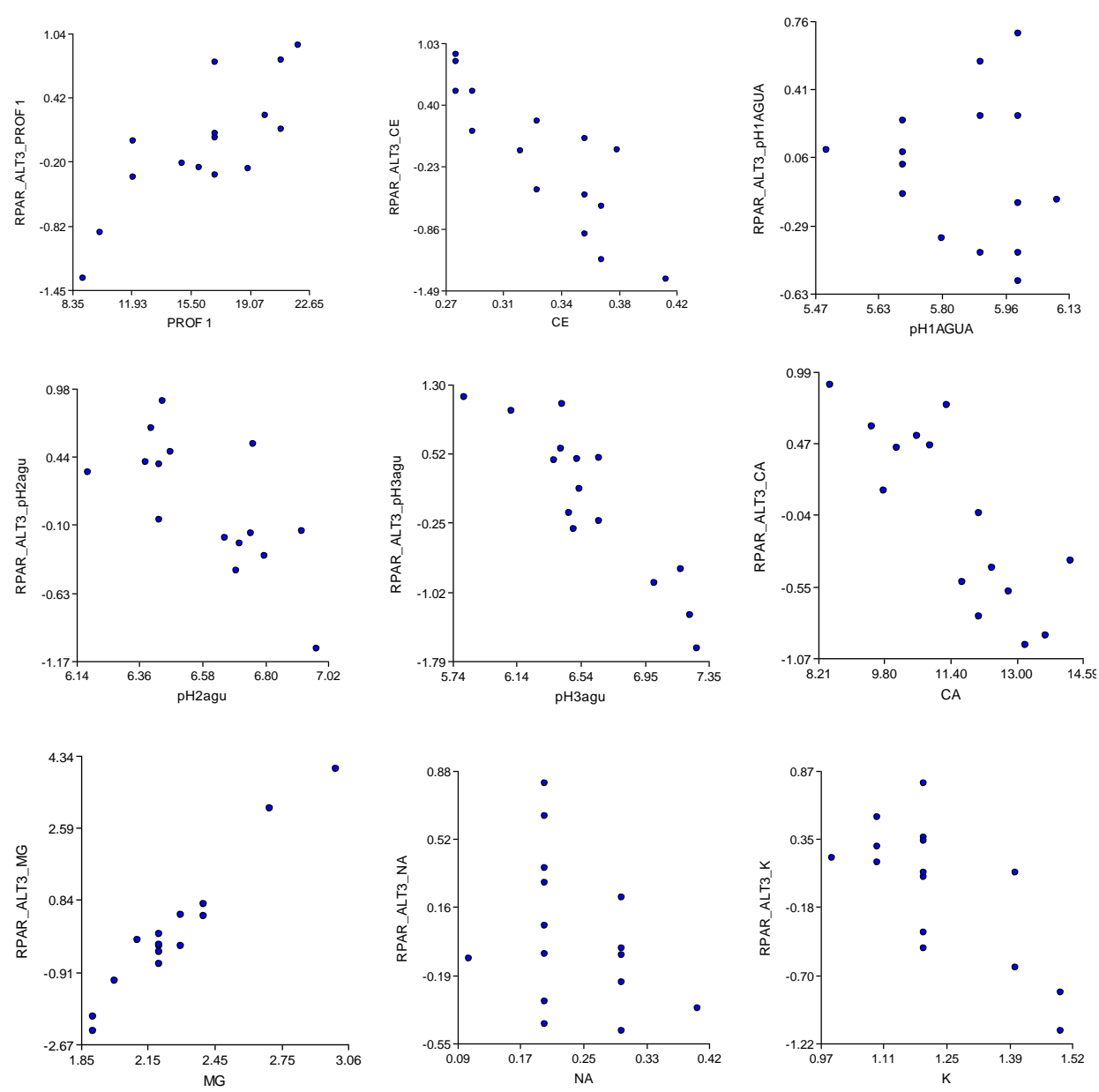

$\mathrm{pH} 1=\mathrm{pH}$ del horizonte superficial, $\mathrm{pH} 2=\mathrm{pH}$ del horizonte $\mathrm{B}$ y $\mathrm{pH} 3=\mathrm{pH}$ del horizonte $\mathrm{B}_{\mathrm{t}}$

Figura $N !^{\circ} 4$

GRÁFICO DE RESIDUOS DE REGRESIÓN LINEAL MÚLTIPLE DE LA ALTURA TOTAL VERSUS VARIABLES DE SITIO EN ÁRBOLES DOMINANTES SUBMUESTRA 


\section{DISCUSIÓN Y CONCLUSIONES}

Este trabajo evidencia que el crecimiento de los árboles es afectado por atributos edáficos a nivel de micrositio, cobrando importancia las variables químicas y sumando al espesor de horizontes, el contenido de carbono orgánico y los $\mathrm{pH}$ de los horizontes.

Para E. camaldulensis, el $\mathrm{pH}$ del horizonte $\mathrm{B}_{\mathrm{t}}$ fue el atributo edáfico de mayor importancia en relación al crecimiento de los árboles. Esto coincide con lo encontrado por Aparicio y López (1995). Resultados similares se encontraron en E. camaldulensis en Nigeria, donde el pH representó el $38 \%$ de la variación en productividad (Buckley 1987). En contraposición, Mushaka (1998) correlacionó parámetros químicos del suelo y la productividad de $E$. camaldulensis, no encontrando correlaciones significativas para el $\mathrm{pH}$, contenido de materia orgánica y niveles de fósforo y potasio.

Barnes et al. (1998), afirmaron que el crecimiento potencial de los árboles es afectado por la cantidad de suelo ocupado por las raíces de los mismos y por la disponibilidad de agua y nutrientes en este espacio de suelo limitado, siendo la disponibilidad de nutrientes afectada directamente por el $\mathrm{pH}$ del suelo. La reacción del suelo refleja la bondad del ambiente edáfico, tanto para las raíces como para los organismos del suelo; con valores de $\mathrm{pH}$ menores a 4,5 disminuye la actividad biológica y por ende el crecimiento radical. Se producen engrosamientos y otras deformaciones en las raíces, casi siempre como producto de la acción tóxica del Aluminio intercambiable. Los procesos de nitrificación y fijación biológica de Nitrógeno se ven disminuidos o inhibidos (Sutton, 1991). La reacción del suelo también tiene influencia en la disponibilidad y absorción de nutrientes por las plantas así como también la actividad microbiana, siendo un útil indicador de las propiedades químicas de los suelos y del crecimiento de las plantas (USDA, 1999; Bickelhaupt, 2003).

Otros autores encontraron que tanto las variables físicas como químicas estaban relacionadas con el crecimiento de los árboles (Hunter y Gibson, 1984; Carter y Klinka, 1992).

Al analizar una submuestra de árboles dominantes se encontró que los árboles dominantes crecieron en los micrositios con menores $\mathrm{pH}$ del horizonte $\mathrm{B}_{\mathrm{t}}$, menor $\mathrm{CE}$, mayor profundidad del horizonte superficial A y mayor contenido de magnesio.

Si bien, en el análisis discriminante, la categoría social de árboles co-dominantes tuvo un comportamiento similar a los dominantes, los primeros presentan una distribución espacial más homogénea (menor rango) respecto al $\mathrm{pH}$ del horizonte $\mathrm{B}_{\mathrm{t}}$. En ese sentido, Aparicio y López (1995), Harper et al. (2000) y Ligier et al. (2000), encontraron fuertes correlaciones entre las variables de crecimiento y los parámetros físico-químicos de los suelos, donde los árboles dominantes se asociaron a suelos más fértiles y profundos. La capacidad predictiva de los modelos de regresión múltiple mejoró con el uso de la categoría social de los arboles dominantes. Además, esta categoría es la que manifiesta mayor permanencia en los rodales.

Los atributos edáficos pueden ser una alternativa útil para predecir el crecimiento de rodales jóvenes. A escala de micrositio, en E. camaldulensis, el $\mathrm{pH}$ del horizonte $\mathrm{B}_{\mathrm{t}}$ resultó ser el atributo edáfico clave respecto del crecimiento de los árboles analizando todo el rodal.

Si solo se utiliza una submuestra de árboles dominantes en el análisis, el poder predictivo de los modelos mejora sustancialmente y el $\mathrm{pH}$ del horizonte $\mathrm{B}_{\mathrm{t}}$, el espesor del horizonte superficial A, la conductividad eléctrica y el contenido de Magnesio también cobran importancia respecto al crecimiento de los árboles. 
La disminución en el crecimiento en micrositios de $\mathrm{pH}$ alcalino, sugiere que estas especies podrían ser plantadas en suelos acidos ( $\mathrm{pH}$ 5-7) como recomienda Symondsa et al. (2001).

Futuros estudios podrían ser conducidos para analizar el efecto del $\mathrm{pH}$ en la disponibilidad y ciclo de nutrientes, su relación con los nutrientes foliares en los micrositios y su impacto relativo en el crecimiento de los árboles.

\section{REFERENCIAS}

Aparicio, J. L. y López, J. A., 1995. Informe Técnico № 7. Potencial de Eucalyptus grandis en los suelos del sudeste de la provincia de Corrientes y algunos factores edáficos relacionados con la producción de madera. Simposio IUFRO para el Cono Sur Sudamericano. 25-30 abril de 1995. Valdivia. Chile.pp 93-108.

Baridon, J. E., Lanfranco, J. W., Marlats, R. M. y Vazquez, M., 2001. Evaluacion de la calidad de sitio forestal para Eucalyptus camaldulensis mediante Indices Edaficos en Argiudoles y Argiacuoles en Argentina. Agricultura Tecnica Chile: 61:2 (192-201).

Barnes, B., Zak, D., Denton, S. and Spurr, S., 1998. Forest Ecology. John Wiley \& Sons, Inc.

Bickelhaupt, Donald, 2003. Instructional Support Specialist, Faculty of Forest and Natural Resources Management. En: www.esf.edu/pubprog/brochure/soilpH

Binkley, D., 1993. Nutrición Forestal. Prácticas de Manejo. Editorial Limusa - Grupo Noriega Editores. (4)

Borzone H.A., Bardi J.F. y Laddaga, J.E., 2007. Crecimiento de Eucalyptus camaldulensis DEHNH cultivado como cortina en un establecimiento agropecuario en el partido de Azul (Pcia. de Buenos Aires). Quebracho 014: 65-74. Universidad Nacional de Santiago del Estero.

Buckley, G. P., 1987. Soil Factors affecting yields of Eucalyptus camaldulensis on former tin-mining land on the Jos Plateau. Interim Report N 12, Dept. of Geography, University of Durham (UK) and Dept. of Geography and Planning, University of Jos, Nigeria.

Carmean, W., 1975. Forest site quality evaluation in United States. Advances in Agronomy 2: 209-289.

Carter, R.E. y Klinka, K., 1992. Variations in shade tolerance of Douglas Fir, Wester Hemlock and Western Redcedar in coastal British Columbia. For.Ecol. Manage.55:87-105.

Correa Neto, T. A., Cunha Dos Anjos, L. H., Pereira, M. G., Amorim H. B. y De Siqueira Jaccoud, C. F., 2007. Ciencia Florestal, Santa Maria, v17, n1, 43-51.

FAO, 1956. Estudios de silvicultura en la repoblación forestal. Roma. 431 pp.

Harper, R. J., Edwards, J. G. and Mc Grath, J .F., 2000. Relating the growth of Tasmanian Blue Gum to site conditions in South Western Australia. Climate Change Newsletter vol.12 nro.1. Febr.

Hunter I. R. and Gibson A. R., 1984. Predicting Pinus radiata site index from environmental variables. New Zealand of Forestry Science 14: 53-64.

INFOSTAT, 2009. InfoStat versión 2009. Grupo InfoStat, FCA, Universidad Nacional de Córdoba, Argentina.

Ligier H. D., Gimenez, L., Kurtz, D., Lopez, J. y Perucca, R., 2000. Relaciones entre Índice de crecimiento medio anual y propiedades de los suelos, en montes forestales de Eucalyptus grandis y Pinus elliottii, en la cuenca del Río Uruguay. Corrientes. Argentina. Universidad Nacional del Nordeste. Comunicaciones Científicas y Tecnológicas. 
Moschini, R., Conti, H. A., Alonso, M., Rodriguez Traverso, J., Nakama, V. y Alfieri, A., 2000. Delimitación de áreas de aptitud climática para el cultivo de eucaliptos en la región Pampeana. PIA 17/96. SAGPyA Forestal No 15. Junio 2000. Argentina. Pag. 2-11.

Mushaka, A., 1998. Relationships between growth, mineral nutrition and site factors for Eucalyptus camaldulensis Dehnh planted in some communal areas of Zimbabwe. Southern African Forestry Journal 183:5965.

Sánchez Acosta, M. y VERA, L., 2005. Situación Foresto-Industrial de Argentina al 2005. III Simposio Iberoamericano de Gestión y Economía Forestal, Ubatuba, Sao Pablo, Brasil.

Sutton, R F., 1991. Soil properties and root development in forest trees: A review. Forestry Canada Ontario Region Greats Lakes Forestry Centre. Information report 0-X-413.

Symondsa, W. L., Campbella, L. C. and Clemensa, J., 2001. Response of ornamental Eucalyptus from acidic and alkaline habitats to potting medium $\mathrm{pH}$. Scientia Horticulturae 88: 121-131.

USDA, 1999. Guía para la Evaluación de la Calidad y Salud del Suelo. Departamento de Agricultura. Servicio de Investigación Agrícola. Servicio de Conservación de Recursos Naturales. Instituto de Calidad de Suelos.

XLSTAT ${ }^{\circledR}$, 2010. Versión 3.05. Addinsoft. En: www.xlstat.com 
